\title{
Global Existence and Boundedness of Solutions to a Chemotaxis-Consumption Model with Singular Sensitivity
}

\author{
Johannes Lankeit $^{1}$ (D) Giuseppe Viglialoro $^{2}$ (D)
}

Received: 23 May 2018 / Accepted: 27 May 2019

(C) Springer Nature B.V. 2019

Abstract In this paper we study the zero-flux chemotaxis-system

$$
\left\{\begin{array}{l}
u_{t}=\Delta u-\chi \nabla \cdot\left(\frac{u}{v} \nabla v\right) \\
v_{t}=\Delta v-f(u) v
\end{array}\right.
$$

in a smooth and bounded domain $\Omega$ of $\mathbb{R}^{2}$, with $\chi>0$ and $f \in C^{1}(\mathbb{R})$ essentially behaving like $u^{\beta}, 0<\beta<1$. Precisely for $\chi<1$ and any sufficiently regular initial data $u(x, 0) \geq 0$ and $v(x, 0)>0$ on $\bar{\Omega}$, we show the existence of global classical solutions. Moreover, if additionally $m:=\int_{\Omega} u(x, 0) d x$ is sufficiently small, then also their boundedness is achieved.

Keywords Nonlinear parabolic systems $\cdot$ Chemotaxis $\cdot$ Singular sensitivity · Global existence $\cdot$ Boundedness

Mathematics Subject Classification (2010) 35Q92 - 35A01 · 35K55 · 35K51 - 92C17

\section{Introduction and Motivations}

Chemotaxis systems in the form of the classical Keller-Segel system [10, 8, 1] model aggregation phenomena in situations where cells are attracted by a signal they themselves emit. If they, instead, direct their movement in response to a substance they consume, the equation governing evolution of the signal concentration becomes much more amenable to providing uniform bounds on this concentration (although, in the most commonly used form, the derivation of bounds for its gradient is more negatively affected by a nonlinearity). If $u$ is

\footnotetext{
$凶$ J. Lankeit

jlankeit@math.uni-paderborn.de

1 Institut für Mathematik, Universität Paderborn, Warburger Str. 100, 33098 Paderborn, Germany

2 Dipartimento di Matematica e Informatica, Università di Cagliari, V. le Merello 92, 09123 Cagliari, Italy
} 
used to denote the population density of these cells and $v$ stands for the concentration of the chemical signal, motion of the cells is governed by the equation

$$
u_{t}=\Delta u-\nabla \cdot(u \nabla S(v))
$$

with some so-called sensitivity function $S$, as in the classical Keller-Segel system (where often $S(v) \equiv \chi v$ is considered), so that cells move in the direction of the gradient of $S(v)$. The signal also diffuses and is consumed (with a rate $f(u)$ that is the higher the more cells are present), i.e.

$$
v_{t}=\Delta v-f(u) v .
$$

Such systems have extensively been studied throughout the past few years, especially in the context of chemotaxis-fluid models (e.g. [5, 17]), and the interested reader can find pointers to the rich literature for example in the introduction of [4].

However, a new difficulty arises if such consumptive chemotaxis models incorporate the effect that small changes in a stimulus affect the response of a biological agent more heavily at a low signal level than the same changes would in presence of high signal concentrations (the so-called 'Weber-Fechner law of stimulus perception', see [11,23]) in the way that the chemotactic sensitivity function is chosen singular, as in

$$
\left\{\begin{array}{l}
u_{t}=\Delta u-\chi \nabla \cdot\left(\frac{u}{v} \nabla v\right) \\
v_{t}=\Delta v-u v
\end{array}\right.
$$

where small signal concentrations enhance the possibly destabilizing cross-diffusive contribution of the chemotaxis term in the first equation.

This system goes back to Keller and Segel studying the formation of travelling bands of E. coli [11]. (For more results on travelling wave solutions in this and related models, see [20].) With respect to global existence of solutions it has been less extensively studied than its signal-production relative

$$
\left\{\begin{array}{l}
u_{t}=\Delta u-\chi \nabla \cdot\left(\frac{u}{v} \nabla v\right) \\
v_{t}=\Delta v-v+u
\end{array}\right.
$$

for recent studies of which we refer to $[7,15]$ and the references therein. As to the occurrence of blow-up that might be expected in this type of equations, the closest result we are aware of is [18], showing blow-up for solutions to a parabolic-elliptic variant of (2) in dimensions 3 and higher for large $\chi$. So far, blow-up has not been proven to occur in (1).

Nevertheless, it is known that (1) admits global solutions if posed in $\mathbb{R}^{2}$ or $\mathbb{R}^{3}$, under a smallness condition on the initial data $\left(u_{0}, v_{0}\right)$, involving $H^{2}$-norms of $u_{0}$ and $\nabla v_{0}$, [22]. In bounded, convex two-dimensional domains it is known from [26] that for arbitrarily large initial data, global solutions exist in a generalized sense. Moreover, they become eventually smooth if the initial mass $\int_{\Omega} u_{0}$ is sufficiently small [24]. (The same article [24] also identifies a smallness condition on $\left(u_{0}, \nabla v_{0}\right)$ in $L \log L(\Omega) \times L^{2}(\Omega)$ which leads to global existence of classical solutions.) Similar results were achieved for a fluid-coupled variant of (1) in [21] and [3]. In the three-dimensional setting, however, the smallness condition in [22] or, instead, restriction to the setting of radial symmetry and renormalized solutions [27] seem to be necessary for all known proofs of global existence.

Modifications that ensure global existence of solutions are using nonlinear diffusion of porous medium type (that is, replacing $\Delta u$ by $\Delta u^{m}$ ), which guarantees global existence (in 
bounded domains of $\mathbb{R}^{n}$ ) as long as $m>1+\frac{n}{4}$, [13], or weakening the cross-diffusive term by replacing $\frac{u}{v} \nabla v$ by, essentially, an expression of the form $\frac{u^{\alpha}}{v} \nabla v$ for $\alpha<1-\frac{n}{4}$, [16].

Apart from these rather strong changes to the diffusive parts of the system, currently it seems that the two-dimensional case of (1) is just barely out of reach for global existence assertions concerning classical solutions emanating from rather general initial data, as witnessed by the fact that eventually smooth weak solutions exist (see above) or by recent results on how the presence of logistic source terms (i.e. if $+\kappa u-\mu u^{2}$ is added to the right-hand side of the first equation in (2)) affects global solvability [14]: While in higher dimensions, global classical solvability results from $\mu$ being sufficiently large, in bounded domains $\Omega \subset \mathbb{R}^{2}$, any $\mu>0$ suffices, provided that $\chi<\sqrt{\frac{2}{n}}$, a number that also plays a role for global existence of solutions to (2) (see [6]). Furthermore, sources with stronger absorption, $+\kappa u-\mu u^{\alpha}, \alpha>1+\frac{n}{2}$, can ensure global existence, [28].

It can be expected that also lessening the impact of high values of the first solution component on the evolution of the second should enforce global existence of solutions. But by how much does it have to be lessened? What happens if the signal substance is consumed with a rate sublinearly depending on the bacterial density?

Indeed, we will show that, at least for $\chi<\sqrt{\frac{2}{n}}=1$, any sublinear dependence of the consumption term on $u$ immediately suffices for globally existent classical solutions. Those will, moreover, remain bounded, if additionally the (initial) mass of bacteria is small.

\section{Main Result and Structure of the Paper}

In agreement with all of the above, this paper is dedicated to the following problem

$$
\begin{cases}u_{t}=\Delta u-\chi \nabla \cdot\left(\frac{u}{v} \nabla v\right) & \text { in } \Omega \times(0, \infty), \\ v_{t}=\Delta v-f(u) v & \text { in } \Omega \times(0, \infty), \\ \frac{\partial u}{\partial v}=\frac{\partial v}{\partial v}=0 & \text { in } \partial \Omega \times(0, \infty), \\ u(x, 0)=u_{0}(x) \quad \text { and } \quad v(x, 0)=v_{0}(x), & x \in \Omega\end{cases}
$$

defined in a bounded and smooth domain $\Omega$ of $\mathbb{R}^{2}$ and with $0<\chi<1$, where the function $f$ relating the amount of bacteria and the rate of signal consumption generalizes a sublinear power-law in that it satisfies

$$
f \in C^{1}(\mathbb{R}) \quad \text { and } \quad 0 \leq f(s) \leq s^{\beta} \quad \text { for all } s>0
$$

and, occasionally,

$$
0 \leq f^{\prime}(s) \leq \beta s^{\beta-1} \text { for all } s>0
$$

for some $0<\beta<1$, and where

$$
\left(u_{0}, v_{0}\right) \in C^{0}(\bar{\Omega}) \times W^{1, r}(\Omega) \text { for some } r>2 \text {, satisfying } u_{0} \geq 0 \text { and } v_{0}>0 \text { in } \bar{\Omega},
$$

are the initial distribution of cells and chemical concentration. Moreover, the zero-flux boundary conditions on both $u$ and $v$ model that the domain is totally insulated.

Under these assumptions we will show that classical solutions exist globally: 
Theorem 2.1 (Global existence) Let

$$
\left\{\begin{array}{l}
\Omega \subset \mathbb{R}^{2} \text { be a smooth and bounded domain, } \\
\beta, \chi \in(0,1) \\
f \text { satisfy (4). }
\end{array}\right.
$$

Then for any given $\left(u_{0}, v_{0}\right)$ as in (6), there is a unique pair of functions $(u, v)$,

$$
\left\{\begin{array}{l}
u \in C^{0}(\bar{\Omega} \times[0, \infty)) \cap C^{2,1}(\bar{\Omega} \times(0, \infty)), \\
v \in C^{0}(\bar{\Omega} \times[0, \infty)) \cap C^{2,1}(\bar{\Omega} \times(0, \infty)) \cap L_{l o c}^{\infty}\left([0, \infty), W^{1, r}(\Omega)\right),
\end{array}\right.
$$

which solve problem (3).

Moreover, if additional smallness assumptions are imposed on the initial bacterial mass (an also biologically meaningful quantity), we can assert boundedness of these solutions.

Theorem 2.2 (Boundedness) Let (A) and (5) be satisfied. Then, it is possible to find a positive $m_{*}$ with the property that for any given $\left(u_{0}, v_{0}\right)$ as in (6) and such that $\int_{\Omega} u_{0}(x) \leq m_{*}$, there is a unique pair of functions $(u, v)$ as in (7) which solve problem (3) and are bounded in $\Omega \times(0, \infty)$.

Remark 2.3 Since blow-up results for (3) are not yet available, we cannot say whether (4), (5) or the condition on $\chi$ are sharp. With small adaptations (for details see Remark 4.2), it will, however, be possible to recover global existence of solutions with small initial mass in the case of $\beta=1$, known from [24].

The next section, Sect. 3, will mainly be concerned with a local-in-time existence result. In particular, we show (in Lemma 3.5) that controlling

$$
\|w(\cdot, t)\|_{L^{\infty}(\Omega)}, \int_{\Omega} u(\cdot, t) \log u(\cdot, t), \quad \text { and } \quad \int_{0}^{t} \int_{\Omega} \frac{|\nabla u|^{2}}{u}
$$

for $t \in[0, T)$, where $w:=-\log \left(\frac{v}{\left\|v_{0}\right\|_{L^{\infty}(\Omega)}}\right)$ arises from the common transformation (see e.g. $[26,13])$ which serves to replace $-\frac{\nabla v}{v}$ by the nonsingular $\nabla w$, is sufficient for the conclusion that the solution exists longer than merely up to time $T$.

In Sect. 4, we then set out to derive bounds on these quantities in order to assert global existence of the previously found local solutions, and hence prove Theorem 2.1. We will achieve this by consideration of the functional

$$
\int_{\Omega} u \log u+a \int_{\Omega} u w
$$

(whose usefulness in similar arguments pertaining to the different system (2) has long been known, see [12, 2], but which appears to be new for consumptive systems).

The functional on whose properties the proof of eventual boundedness in [24] relies, is

$$
\int_{\Omega}\left(u \log u+|\nabla w|^{2}\right)
$$


we now (i.e. in Sect. 5, which is devoted to the proof of Theorem 2.2) treat the similar

$$
\mathcal{G}(t)=\mathcal{G}(u, w)=\frac{1}{2} \int_{\Omega}|\nabla w|^{2}+\int_{\Omega} H(u),
$$

see (36), where $H$ is a second primitive of $\sigma \mapsto \frac{f^{\prime}(\sigma)}{\chi \sigma}$, in order to derive boundedness of $u$ on $\left[t_{0}, \infty\right)$ for some $t_{0}>0$, for small-mass solutions. Boundedness on finite time intervals $\left[0, t_{0}\right)$ is no longer an issue thanks to Theorem 2.1 .

Remark 2.4 Constants in many statements of lemmata and proofs will depend on the total mass $\int_{\Omega} u_{0}$ of bacteria, which we will, throughout the article, denote by $m$. Sometimes (like in Theorem 2.2), we will require smallness of $m$, finally indicated by the condition $m<m_{*}$, where $m_{*}>0$ is a thresholds to be introduced in Lemma 5.9 and in turn depends on the constants $\bar{m}$ and $\hat{m}$ originating from Lemmata 5.7 and 5.8, respectively. Also smallness conditions on an upper bound $M$ for $\int_{\Omega}|\nabla w|^{2}$ (cf. Lemmata 5.6 and 5.7) will be reduced to the requirement $m<m_{*}$ (Lemma 5.9).

\section{Existence of Local-in-Time Solutions and Preparatory Lemmas}

Let us firstly give a result concerning local-in-time existence of classical solutions to system (3).

Lemma 3.1 Assume (A). Then, for any given $\left(u_{0}, v_{0}\right)$ as in $(6)$, there are $T_{\max } \in(0, \infty]$ and a uniquely determined pair of functions $(u, v)$ with regularity as in (7) which solve problem (3) in $\Omega \times\left(0, T_{\max }\right)$ and are such that if $T_{\max }<\infty$ then

$$
\limsup _{t \nearrow T_{\max }}\left(\|u(\cdot, t)\|_{L^{\infty}(\Omega)}+\|v(\cdot, t)\|_{W^{1, r}(\Omega)}\right)=\infty .
$$

Moreover, we have

$$
\int_{\Omega} u(\cdot, t)=m=\int_{\Omega} u_{0} \quad \text { for all } t \in\left(0, T_{\max }\right),
$$

and

$$
u \geq 0 \quad \text { and } \quad 0<v \leq\left\|v_{0}\right\|_{L^{\infty}(\Omega)} \quad \text { in } \bar{\Omega} \times\left(0, T_{\max }\right)
$$

Proof The claim concerning the local existence and uniqueness as well as the extensibility criterion (8) can be shown by straightforward adaptations of well-established methods involving an appropriate fixed point framework and standard parabolic regularity theory (see, for instance, [26, Lemma 2.2] or [1, Lemma 3.1]).

On the other hand, taking into consideration the no-flux boundary conditions for problem (3), an integration of its first equation over $\Omega$ provides

$$
\frac{d}{d t} \int_{\Omega} u=0 \quad \text { for all } t \in\left(0, T_{\max }\right),
$$

so that $\int_{\Omega} u=\int_{\Omega} u_{0}=m$ and (9) is shown.

Since $u_{0} \geq 0$ and $v_{0}>0$, comparison arguments apply to yield both expressions in (10). 
Once the local existence of solutions to (3) is attained, through the transformation

$$
w:=-\log \left(\frac{v}{\left\|v_{0}\right\|_{L^{\infty}(\Omega)}}\right), \quad w_{0}:=-\log \left(\frac{v_{0}}{\left\|v_{0}\right\|_{L^{\infty}(\Omega)}}\right),
$$

which has already been used in [13] and [26], we get that $w \geq 0$ in $\Omega \times\left(0, T_{\max }\right)$, and that $(u, w) \in\left(C^{0}\left(\bar{\Omega} \times\left[0, T_{\max }\right)\right) \cap C^{2,1}\left(\bar{\Omega} \times\left(0, T_{\max }\right)\right)\right)^{2}$ also (classically) solves the transformed problem

$$
\begin{cases}u_{t}=\Delta u+\chi \nabla \cdot(u \nabla w) & \text { in } \Omega \times\left(0, T_{\text {max }}\right), \\ w_{t}=\Delta w-|\nabla w|^{2}+f(u) & \text { in } \Omega \times\left(0, T_{\max }\right), \\ \frac{\partial u}{\partial \nu}=\frac{\partial w}{\partial \nu}=0 & \text { in } \partial \Omega \times\left(0, T_{\max }\right), \\ u(x, 0)=u_{0}(x) \geq 0 \quad w(x, 0)=w_{0}(x) \geq 0, & x \in \bar{\Omega}\end{cases}
$$

It can be seen that in this last system the first equation does not present the singularity at $v=0$ appearing in (3), so that this version will be considered in some places in this paper. This system resembles the classical Keller-Segel system, apart from a change of the sign in the first equation (which is mathematically disadvantageous with regards to arguments relying on an energy structure) and the additional term $-|\nabla w|^{2}$ in the second equation. Note that the "good sign" of this additional term is of little help, since dealing with the contribution of $w$ to the first equation requires some gradient bounds.

Let us also recall those special cases of the well-known Gagliardo-Nirenberg inequality which will be used through the paper to prove the main theorems.

Lemma 3.2 (Gagliardo-Nirenberg inequality) Let $\Omega$ be a bounded Lipschitz domain of $\mathbb{R}^{2}$. Then there is a constant $C_{G N}>0$ such that the following inequalities hold: With $\mathfrak{q}, \mathfrak{s} \in\{1,2\}$, $\mathfrak{p} \in[2,4], \theta=1-\frac{\mathfrak{q}}{\mathfrak{p}} \in[0,1)$,

$$
\|f\|_{L^{\mathfrak{p}}(\Omega)} \leq C_{G N}\left(\|\nabla f\|_{L^{2}(\Omega)}^{\theta}\|f\|_{L^{\mathfrak{q}}(\Omega)}^{1-\theta}+\|f\|_{L^{\mathfrak{s}}(\Omega)}\right)
$$

is satisfied for all $f \in L^{\mathfrak{q}}(\Omega)$ with $\nabla f \in L^{2}(\Omega)$,

$$
\|f\|_{L^{3}(\Omega)} \leq C_{G N}\|f\|_{L^{1}(\Omega)}^{\frac{1}{3}}\|f\|_{W^{1,2}(\Omega)}^{\frac{2}{3}} \quad \text { for all } f \in W^{1,2}(\Omega) .
$$

Finally, for any $f \in W^{2,2}(\Omega)$ fulfilling $\frac{\partial f}{\partial v}=0$ on $\partial \Omega$ we have that

$$
\|\nabla f\|_{L^{4}(\Omega)}^{4} \leq C_{G N}\|\nabla f\|_{L^{2}(\Omega)}^{2}\|\Delta f\|_{L^{2}(\Omega)}^{2} .
$$

Proof See [19].

In order to avoid convexity conditions on the domain, let us recall the following estimate:

Lemma 3.3 Let $\Omega \subset \mathbb{R}^{n}, n \geq 1$, be a bounded domain with smooth boundary. Then there is $C_{\partial \Omega}>0$ such that for every function $f \in C^{2}(\bar{\Omega})$ with $\frac{\partial f}{\partial v}=0$ on $\partial \Omega$, the inequality

$$
2 \int_{\partial \Omega}|\nabla f|^{2} \frac{\partial|\nabla f|^{2}}{\partial v} \leq\left.\left.\frac{1}{16} \int_{\Omega}|\nabla| \nabla f\right|^{2}\right|^{2}+C_{\partial \Omega}\left(\int_{\Omega}|\nabla f|^{2}\right)^{2}
$$

holds. 
Proof A proof can be found in [9, Prop. 3.2]. It is based on embeddings of the form $W^{r+\frac{1}{2}, 2}(\Omega) \hookrightarrow L^{2}(\partial \Omega)$ for $r \in\left(0, \frac{1}{2}\right)$ and a Gagliardo-Nirenberg inequality for fractional Sobolev spaces, combined with estimates of $\frac{\partial|\nabla w|^{2}}{\partial v}$ on $\partial \Omega$. For convex domains, the left side actually is nonpositive.

The following result will enable us to estimate the spatio-temporal $L^{2}$-norm of the cells' density by their initial mass.

Lemma 3.4 Let $\Omega \subset \mathbb{R}^{2}$ be a smooth and bounded domain, let $T>0, c_{1}, c_{2}>0$ and $m>0$. Then every function $u \in C^{0}(\bar{\Omega} \times[0, T)) \cap C^{2,1}(\bar{\Omega} \times(0, T))$ which fulfils

$$
\int_{\Omega} u(\cdot, t)=m \quad \text { and } \quad \int_{0}^{t} \int_{\Omega} \frac{|\nabla u|^{2}}{u} \leq c_{1} t+c_{2} \quad \text { for all } t \in(0, T)
$$

for all $t \in(0, T)$ also satisfies

$$
\int_{0}^{t} \int_{\Omega} u^{2} \leq C_{1}\left(m, c_{1}\right) t+C_{2}\left(m, c_{2}\right)
$$

where

$$
C_{1}\left(m, c_{1}\right):=m\left(2 C_{G N}\right)^{4}\left(c_{1}+m\right), \quad C_{2}\left(m, c_{2}\right):=\left(2 C_{G N}\right)^{4} m c_{2} .
$$

Proof The Gagliardo-Nirenberg inequality (13) with $\mathfrak{p}=4, \mathfrak{q}=\mathfrak{s}=2$ and $\theta=\frac{1}{2}$, together with

$$
(A+B)^{k} \leq 2^{k}\left(A^{k}+B^{k}\right)
$$

valid for any $A, B \geq 0$ and $k>0$, enables us to estimate

$$
\begin{aligned}
\int_{\Omega} u^{2} & =\|\sqrt{u}\|_{L^{4}(\Omega)}^{4} \\
& \leq\left[C_{G N}\left(\|\nabla \sqrt{u}\|_{L^{2}(\Omega)}^{\theta}\|\sqrt{u}\|_{L^{2}(\Omega)}^{1-\theta}+\|\sqrt{u}\|_{L^{2}(\Omega)}\right)\right]^{4} \\
& \leq\left(2 C_{G N}\right)^{4}\left[m\left(\int_{\Omega} \frac{|\nabla u|^{2}}{u}\right)+m^{2}\right] \quad \text { on }(0, T),
\end{aligned}
$$

and so for $t \in(0, T)$, thanks to the assumption on $\int_{0}^{t} \int_{\Omega} \frac{|\nabla u|^{2}}{u}$, we have that (16) holds.

As a first application of Lemma 3.4, let us sharpen the extensibility criterion (8).

Lemma 3.5 Let $\Omega$ be a smooth and bounded domain of $\mathbb{R}^{2}$ and $\chi \geq 0, \beta \in(0,1]$ and let $f$ be as in (4). For any given $\left(u_{0}, v_{0}\right)$ as in $(6)$, let $(u, v)$ be the local-in-time classical solution of problem (3) provided by Lemma 3.1, and $(u, w)$ that of the transformed problem (12), $w$ being the function introduced in (11). If there exists a positive constant $C$ such that for all $t \in\left(0, T_{\max }\right)$

$$
\left\{\begin{array}{l}
\int_{0}^{t} \int_{\Omega} \frac{|\nabla u|^{2}}{u} \leq C(1+t) \\
\int_{\Omega} u(\cdot, t) \log u(\cdot, t) \leq C(1+t) \\
\|w(\cdot, t)\|_{L^{\infty}(\Omega)} \leq C(1+t)
\end{array}\right.
$$

then $T_{\max }=\infty$. 
Proof We will, to the contrary, assume $T_{\max }$ finite, and derive a contradiction to (8). Let $m=\int_{\Omega} u=\int_{\Omega} u_{0}$, as in Lemma 3.1. Recalling (11), we have that

$$
\frac{1}{v}=\frac{e^{w}}{\left\|v_{0}\right\|_{L^{\infty}(\Omega)}}
$$

and the third assumption in (18) warrants that with some $L>0$

$$
\frac{1}{v} \leq L \quad \text { in } \Omega \times\left(0, T_{\max }\right)
$$

Now, from the estimate in Lemma 3.4, we can deduce also some bound of $\int_{\Omega}|\nabla v|^{2}$ on $\left(0, T_{\max }\right)$. In fact, a differentiation in time and the Young inequality allow us, through the second equation of (3) and by estimate (4), to get

$$
\begin{aligned}
\frac{d}{d t} \int_{\Omega}|\nabla v|^{2} & =2 \int_{\Omega} \nabla v \cdot \nabla v_{t}=2 \int_{\Omega} \nabla v \cdot(\nabla \Delta v-\nabla(f(u) v)) \\
& =-2 \int_{\Omega}(\Delta v)^{2}+2 \int_{\Omega}(f(u) v) \Delta v \\
& \leq-2 \int_{\Omega}(\Delta v)^{2}+\int_{\Omega}(\Delta v)^{2}+\int_{\Omega} u^{2 \beta} v^{2} \quad \text { in }\left(0, T_{\max }\right) .
\end{aligned}
$$

Moreover, again from $\beta<1$, the Young inequality and (10) we infer

$$
\int_{\Omega} u^{2 \beta} v^{2} \leq\left\|v_{0}\right\|_{L^{\infty}(\Omega)}^{2} \beta \int_{\Omega} u^{2}+\left\|v_{0}\right\|_{L^{\infty}(\Omega)}^{2}(1-\beta)|\Omega| \quad \text { in }\left(0, T_{\max }\right),
$$

so that, neglecting the nonpositive term $-\int_{\Omega}(\Delta v)^{2}$ we obtain

$$
\frac{d}{d t} \int_{\Omega}|\nabla v|^{2} \leq\left\|v_{0}\right\|_{L^{\infty}(\Omega)}^{2} \beta \int_{\Omega} u^{2}+\left\|v_{0}\right\|_{L^{\infty}(\Omega)}^{2}(1-\beta)|\Omega| \quad \text { in }\left(0, T_{\max }\right) .
$$

Finally, by means of (16), an integration over $(0, t)$ yields, for

$$
C_{2}=\left\|v_{0}\right\|_{L^{\infty}(\Omega)}^{2}\left(\beta C_{1}+(1-\beta)|\Omega|\right),
$$

that

$$
\int_{\Omega}|\nabla v(\cdot, t)|^{2} \leq C_{2}(1+t) \quad \text { for all } t \in\left(0, T_{\max }\right) .
$$

Having derived these bounds, we will next attempt to reduce the present problem to the setting of the standard extensibility criterion of [1, Sect. 3]. According to some ideas used in [12], for the positive constant $L$ above introduced, let $\xi_{L}: \mathbb{R} \rightarrow[0,1]$ be a smooth, decreasing function verifying $\xi_{L}(v)=1$ for $v \leq 1 /(2 L)$ and $\xi_{L}(v)=0$ for $v \geq 1 / L$. Subsequently, the function

$$
S(x, t, u, v)=\xi_{L}(v) \frac{2 \chi}{L}+\left(1-\xi_{L}(v)\right) \frac{\chi}{v}, \quad(x, t, u, v) \in \bar{\Omega} \times[0, \infty) \times \mathbb{R}^{2},
$$

belongs to $C_{\text {loc }}^{1+\omega}\left(\bar{\Omega} \times[0, \infty) \times \mathbb{R}^{2}\right)$, for some $\omega \in(0,1)$, and, additionally, satisfies $S(x, t, u, v) \equiv \frac{\chi}{v}$ for all $v \geq 1 / L$. 
From all of the above and following the nomenclature of [1, Sect. 3], setting

$$
f(x, t, u, v) \equiv 0, \quad \text { and } \quad g(x, t, u, v)=v-f(u) v,
$$

the two partial differential equations of problem (3) read

$$
\begin{cases}u_{t}=\Delta u-\nabla \cdot(u S(x, t, u, v) \nabla v)+f(x, t, u, v) & \text { in } \Omega \times\left(0, T_{\max }\right) \\ v_{t}=\Delta v-v+g(x, t, u, v) & \text { in } \Omega \times\left(0, T_{\max }\right)\end{cases}
$$

by virtue of bound (19). In particular, besides $S \in C_{\text {loc }}^{1+\omega}\left(\bar{\Omega} \times[0, \infty) \times \mathbb{R}^{2}\right)$, we have also that $f \in C_{\mathrm{loc}}^{1-}\left(\bar{\Omega} \times[0, \infty) \times \mathbb{R}^{2}\right)$ and $g \in C_{\mathrm{loc}}^{1-}\left(\bar{\Omega} \times[0, \infty) \times \mathbb{R}^{2}\right)$, as well as $f(x, t, 0, v)=0$ for all $(x, t, v) \in \bar{\Omega} \times[0, \infty) \times \mathbb{R}$ and $g(x, t, u, 0)=0$ for all $(x, t, u) \in \bar{\Omega} \times[0, \infty) \times \mathbb{R}$.

After these preparations, we can conclude that $T_{\max }=\infty$. Indeed, in view of the assumptions in (18), estimate (20), the regularity and boundedness of both $S$ and $v$ and the expression of $g$ given above, there exists a positive $N$ such that for all $t \in\left(0, T_{\max }\right)$

$$
\left\{\begin{array}{l}
\int_{\Omega}|\nabla v(\cdot, t)|^{2} \leq N, \quad \int_{\Omega} u(\cdot, t) \log u(\cdot, t) \leq N \\
S(x, t, u, v) \leq N, \quad|g(x, t, u, v)| \leq N(1+u) .
\end{array}\right.
$$

In such conditions, all the hypotheses of [1, Lemma 3.3] are accomplished and hence the same lemma implies boundedness of $t \mapsto\|u(\cdot, t)\|_{L^{\infty}(\Omega)}+\|v(\cdot, t)\|_{W^{1, r}(\Omega)}$ on $\left(0, T_{\max }\right)$. This contradicts the extensibility criterion (8), and therefore $T_{\max }=\infty$.

\section{Existence of Global Solutions: Proof of Theorem 2.1}

In this section, starting from the local solution $(u, w)$ to problem (12), we define, for some proper $a>0$, whose precise value is to be chosen during the next proof, the following energy functional

$$
\mathfrak{F}=\mathfrak{F}(t)=\mathfrak{F}(u, w):=\int_{\Omega} u \log u+a \int_{\Omega} u w \quad \text { on }\left(0, T_{\max }\right),
$$

and its initial value

$$
\mathfrak{F}(0)=\int_{\Omega} u_{0} \log u_{0}+a \int_{\Omega} u_{0} w_{0} .
$$

An investigation of its time depending behaviour will reveal useful estimates to be employed in the proof of Theorem 2.1.

Lemma 4.1 Assume (A). Let $m>0$. Then there is $L_{1}=L_{1}(m)>0$ such that the following holds: For any given $\left(u_{0}, v_{0}\right)$ as in $(6)$, and additionally satisfying $\int_{\Omega} u_{0}=m$, let $(u, v)$ be the local-in-time classical solution of problem (3) provided by Lemma 3.1, and $(u, w)$ that of the transformed problem (12), $w$ being the function introduced in (11). Then we can find a positive constant $L_{2}$ such that

$$
\int_{\Omega} u(\cdot, t) \log u(\cdot, t) \leq L_{1} t+L_{2} \quad \text { for all } t \in\left(0, T_{\max }\right),
$$

and

$$
\int_{0}^{t} \int_{\Omega} \frac{|\nabla u|^{2}}{u} \leq L_{1} t+L_{2} \quad \text { for all } t \in\left(0, T_{\max }\right) .
$$

Moreover, $L_{1}(m)$ remains bounded in a neighbourhood of $m=0$. 
Proof With some positive $a \in\left(a_{-}, a_{+}\right)$, where $a_{-}$and $a_{+}$will be explicitly computed later, starting from $\mathfrak{F}$ defined in (21), we firstly observe from the inequality $s \log s \geq-\frac{1}{e}$, valid for every $s>0$, and nonnegativity of $u$ and $w$ that

$$
\mathfrak{F}(u, w) \geq-\frac{|\Omega|}{e} \quad \text { on }\left(0, T_{\max }\right) .
$$

In view of (12), a differentiation of $\mathfrak{F}$ and the divergence theorem provide

$$
\begin{aligned}
\frac{d}{d t} \mathfrak{F}= & \int_{\Omega}\left(u_{t} \log u+u_{t}\right)+a \int_{\Omega} u_{t} w+a \int_{\Omega} u w_{t} \\
= & \int_{\Omega} \Delta u \log u+\chi \int_{\Omega} \nabla \cdot(u \nabla w) \log u+a \int_{\Omega} w \Delta u \\
& +a \chi \int_{\Omega} w \nabla \cdot(u \nabla w)+a \int_{\Omega} u \Delta w-a \int_{\Omega} u|\nabla w|^{2}+a \int_{\Omega} u f(u) \\
\leq & -\int_{\Omega} \frac{|\nabla u|^{2}}{u}-(\chi+2 a) \int_{\Omega} \nabla u \cdot \nabla w \\
& -a(\chi+1) \int_{\Omega} u|\nabla w|^{2}+a \int_{\Omega} u^{\beta+1} \text { on }\left(0, T_{\max }\right) .
\end{aligned}
$$

Now, on the one hand the Young inequality implies

$$
\begin{aligned}
-(\chi+2 a) \int_{\Omega} \nabla u \cdot \nabla w \leq & a(\chi+1) \int_{\Omega} u|\nabla w|^{2} \\
& +\frac{(\chi+2 a)^{2}}{4 a(\chi+1)} \int_{\Omega} \frac{|\nabla u|^{2}}{u} \text { on }\left(0, T_{\max }\right),
\end{aligned}
$$

while on the other hand, again the Gagliardo-Nirenberg inequality (13), with $\mathfrak{p}=2(\beta+1)$, $\mathfrak{q}=\mathfrak{s}=2$ and $\theta=\frac{\beta}{\beta+1}$, and (17) control the term $a \int_{\Omega} u^{\beta+1}$ in this form:

$$
\begin{aligned}
\int_{\Omega} u^{\beta+1} & =\|\sqrt{u}\|_{L^{2(\beta+1)}(\Omega)}^{2(\beta+1)} \\
& \leq\left[C_{G N}\left(\|\nabla \sqrt{u}\|_{L^{2}(\Omega)}^{\theta}\|\sqrt{u}\|_{L^{2}(\Omega)}^{(1-\theta)}+\|\sqrt{u}\|_{L^{2}(\Omega)}\right)\right]^{2(\beta+1)} \\
& \leq\left(2 C_{G N}\right)^{2(\beta+1)}\left[m\left(\int_{\Omega} \frac{|\nabla u|^{2}}{u}\right)^{\beta}+m^{\beta+1}\right] \quad \text { on }\left(0, T_{\max }\right) .
\end{aligned}
$$

Now, in view of the assumption $0<\chi<1$, the numbers

$$
a_{-}=\frac{1}{2}-\frac{1}{2} \sqrt{1-\chi^{2}} \text { and } a_{+}=\frac{1}{2}+\frac{1}{2} \sqrt{1-\chi^{2}}
$$

are real, and for any $a \in\left(a_{-}, a_{+}\right)$the constant $c_{0}=1-\frac{(\chi+2 a)^{2}}{4 a(\chi+1)}$ is positive. Hence an application of Young's inequality in (27) shows that on $\left(0, T_{\max }\right)$

$$
\int_{\Omega} u^{\beta+1} \leq \frac{c_{0}}{2 a} \int_{\Omega} \frac{|\nabla u|^{2}}{u}+c_{1} m^{\frac{1}{1-\beta}}+\left(2 C_{G N}\right)^{2(\beta+1)} m^{\beta+1},
$$


where $c_{1}=(1-\beta)\left(2 C_{G N}\right)^{\frac{2(\beta+1)}{1-\beta}}\left(\frac{2 a \beta}{c_{0}}\right)^{\frac{\beta}{1-\beta}}$. By inserting (28) and (26) into (25) and setting $c_{2}(m)=a c_{1} m^{\frac{\beta}{1-\beta}}+a\left(2 C_{G N}\right)^{2(\beta+1)} m^{\beta+1}$ we get

$$
\frac{d}{d t} \mathfrak{F}+\frac{c_{0}}{2} \int_{\Omega} \frac{|\nabla u|^{2}}{u} \leq c_{2}(m) \quad \text { on }\left(0, T_{\max }\right),
$$

and an integration on $(0, t)$, for $t<T_{\max }$, in conjunction with the bound from below of $\mathfrak{F}$ (expression (24)), enables us to arrive at

$$
-\frac{|\Omega|}{e} \leq \mathfrak{F}(t)=\int_{\Omega} u(\cdot, t) \log u(\cdot, t) \leq \mathfrak{F}(0)+c_{2}(m) t \quad \text { for all } t \in\left(0, T_{\text {max }}\right) .
$$

Thereupon, in view of (30), again an integration of (29) on $(0, t)$, with $t<T_{\text {max }}$, leads to

$$
\frac{c_{0}}{2} \int_{0}^{t} \int_{\Omega} \frac{|\nabla u|^{2}}{u} \leq c_{2}(m) t+\mathfrak{F}(0)+\frac{|\Omega|}{e} \text { for } t \in\left(0, T_{\max }\right),
$$

so that (22) and (23) are attained with the choices

$$
L_{1}:=L_{1}(m):=\max \left\{c_{2}(m), \frac{2 c_{2}(m)}{c_{0}}\right\}, \quad L_{2}:=\frac{2}{c_{0}}\left(\mathfrak{F}(0)+\frac{|\Omega|}{e}\right) .
$$

Finally, since $c_{0}, c_{1}$ and $a$ do not depend on $m$, from the expression of $c_{2}(m)$ above we see that $L_{1}(m)$ is bounded close to $m=0$. (More precisely, we even have that $L_{1}(m) \searrow 0$ as $m \searrow 0$.)

Remark 4.2 Even though the functional $\mathfrak{F}$ has not been considered in [24] (nor in [26]), global existence of small-mass solutions in the case of $\beta=1$ (and $\chi<1$ ) can be recovered from the above considerations: If we insert $\beta=1$ into (27) and refrain from using Young's inequality, the remainder of the proof still is applicable, provided that $\left(2 C_{G N}\right)^{4} m<\frac{c_{0}}{2 a}$.

As a consequence of all of the above, we have the necessary ingredients to prove Theorem 2.1.

Proof of Theorem 2.1 Let $(u, w)$ be the local-in-time classical solution of problem (12) provided by Lemma 3.1; clearly the function $w$ also solves

$$
w_{t} \leq \Delta w+f(u) \text { in } \Omega \times\left(0, T_{\max }\right)
$$

and therefore by using a representation formula and (4) we get

$$
w(\cdot, t) \leq e^{t \Delta} w_{0}+\int_{0}^{t} e^{(t-s) \Delta} u^{\beta}(\cdot, s) d s \quad \text { in } \Omega \text { for any } t \in\left(0, T_{\max }\right) .
$$

Now we invoke a standard estimate for the Neumann heat semigroup (see [25, Lemma 1.3]) which warrants the existence of a positive constant $C_{S}$ such that for all $t>0$

$$
\left\|e^{t \Delta} \varphi\right\|_{L^{\infty}(\Omega)} \leq C_{S}\left(1+t^{-\frac{1}{p}}\right)\|\varphi\|_{L^{p}(\Omega)} \quad \text { for all } \varphi \in L^{p}(\Omega)
$$


so that its application with $p=\frac{2}{\beta}$, in conjunction with the Young inequality, provides

$$
\begin{aligned}
\|w(\cdot, t)\|_{L^{\infty}(\Omega)} \leq & \left\|e^{t \Delta} w_{0}\right\|_{L^{\infty}(\Omega)}+\int_{0}^{t}\left\|e^{(t-s) \Delta} u^{\beta}\right\|_{L^{\infty}(\Omega)} d s \\
\leq & \left\|w_{0}\right\|_{L^{\infty}(\Omega)}+C_{S} \int_{0}^{t}\left(1+(t-s)^{-\frac{\beta}{2}}\right)\left(\int_{\Omega} u^{2}\right)^{\frac{\beta}{2}} d s \\
\leq & \left\|w_{0}\right\|_{L^{\infty}(\Omega)}+\frac{C_{S}}{2} \int_{0}^{t}\left(1+(t-s)^{-\frac{\beta}{2}}\right)^{2} d s \\
& +\frac{C_{S}}{2} \int_{0}^{t}\left(\int_{\Omega} u^{2}\right)^{\beta} d s \\
\leq & \left\|w_{0}\right\|_{L^{\infty}(\Omega)}+\frac{C_{S}}{2} t+\frac{C_{S}}{2(1-\beta)} t^{1-\beta}+\frac{2 C_{S}}{2-\beta} t^{\frac{2-\beta}{2}} \\
& +\frac{C_{S}}{2} \beta \int_{0}^{t} \int_{\Omega} u^{2}+\frac{C_{S}}{2}(1-\beta) t, \quad t \in\left(0, T_{\max }\right) .
\end{aligned}
$$

According to Lemmata 3.4 and 4.1, we can write

$$
\int_{0}^{t} \int_{\Omega} u^{2} \leq C_{1}(1+t) \quad t<T_{\max }
$$

where

$$
C_{1}=C_{1}(m)=m\left(2 C_{G N}\right)^{4}\left(L_{1}(m)+m\right),
$$

with $L_{1}(m)>0$ from Lemma 4.1. Thereafter, (31) becomes

$$
\begin{aligned}
\|w(\cdot, t)\|_{L^{\infty}(\Omega)} \leq & \left\|w_{0}\right\|_{L^{\infty}(\Omega)}+\frac{C_{S}}{2} t+\frac{C_{S}}{2(1-\beta)} t^{1-\beta} \\
& +\frac{2 C_{S}}{2-\beta} t^{\frac{2-\beta}{2}}+\frac{C_{S}}{2} \beta C_{1}(1+t)+\frac{C_{S}}{2}(1-\beta) t \\
\leq & L_{3}(m)(1+t), \quad t<T_{\max },
\end{aligned}
$$

where

$$
L_{3}(m):=\frac{C_{S}}{2(1-\beta)}+\frac{C_{S} C_{1} \beta}{2}+\frac{2 C_{S}}{2-\beta}+\max \left\{\left\|w_{0}\right\|_{L^{\infty}(\Omega)}, \frac{C_{S}}{2}+\frac{C_{S}}{2}(1-\beta)\right\} .
$$

Once the bounds (22), (23) and (32) are considered, the conclusion is then a direct consequence of Lemma 3.5 with $C=\max \left\{L_{1}(m), L_{2}, L_{3}(m)\right\}$.

\section{Deriving Boundedness of Global Solutions: Proof of Theorem 2.2}

Now that the global existence of solutions to (3) is guaranteed, let us dedicate this section to the boundedness question: as we shall show in the sequel, this issue will be addressed if some smallness assumption on the initial mass $m$ is given.

In particular, the boundedness of $u$ is achieved by controlling the quantity $\int_{\Omega}|\nabla w|^{p}$ for some $p>2$, as specified in this 
Lemma 5.1 Assuming (A), let $m>0, p>2, K>0$ and $\tau>0$. Then there is a positive constant $C=C(p, m, K, \tau)$ such that for any initial data $\left(u_{0}, v_{0}\right)$ as in (6) with $\int_{\Omega} u_{0}=m$, the solution $(u, w)$ provided by Theorem 2.1 and (11) satisfies the following: If

$$
\int_{\Omega}|\nabla w(\cdot, t)|^{p} \leq K \quad \text { for all } t>t_{0},
$$

holds with some $t_{0} \geq 0$, then

$$
\|u(\cdot, t)\|_{L^{\infty}(\Omega)} \leq C(p, m, K, \tau) \quad \text { for all } t>t_{0}+\tau .
$$

Proof This is [24, Lemma 4.4], since only the first equation of the system is of importance here. We therefore only indicate the main steps, referring to [24, Lemma 4.4] for details. Using that by Hölder's inequality and (33) for $q \in(2, p)$ we have $\|u(\cdot, s) \nabla w(\cdot, s)\|_{L^{q}(\Omega)} \leq$ $m^{1-a} K^{\frac{1}{p}}\|u(\cdot, s)\|_{L^{\infty}(\Omega)}^{a}$ with $a=1-\frac{p-q}{p q}$, from semigroup estimates we can infer that with some $c_{1}, c_{2}>0$

$$
\begin{aligned}
\|u(\cdot, t)\|_{L^{\infty}(\Omega)} & \leq c_{1} m\left(t-t_{0}\right)^{-1}+c_{2} m^{1-a} K^{\frac{1}{p}} \int_{t_{0}}^{t}(t-s)^{-\left(\frac{1}{2}+\frac{1}{q}\right)}\|u(\cdot, s)\|_{L^{\infty}(\Omega)}^{a} d s \\
& \leq c_{1} m\left(t-t_{0}\right)^{-1}+c_{2} m^{1-a} K^{\frac{1}{p}} c_{3} S_{1}^{a}\left(t-t_{0}\right)^{-\frac{p+2}{2 p}}, \quad t \in\left(t_{0}, t_{0}+1\right),
\end{aligned}
$$

where $S_{1}:=\max \left\{\left(t-t_{0}\right)\|u(\cdot, t)\|_{L^{\infty}(\Omega)} \mid t \in\left[t_{0}, t_{0}+1\right]\right\}$ and $c_{3}:=\int_{0}^{1}(1-\sigma)^{-\left(\frac{1}{2}+\frac{1}{q}\right)} \times$ $\sigma^{-a} d \sigma<\infty$. Multiplication of (35) by $\left(t-t_{0}\right)$ shows that we can find an explicit expression of $c_{4}>0$ such that $S_{1} \leq c_{4}$ (see also [24, (4.31)]). For $T>t_{0}$ and $t \in\left[t_{0}+1, T\right.$ ) we similarly derive that

$$
\begin{aligned}
\|u(\cdot, t)\|_{L^{\infty}(\Omega)} & \leq c_{1} m+c_{2} m^{1-a} K^{\frac{1}{p}} \int_{t-1}^{t}(t-s)^{-\left(\frac{1}{2}+\frac{1}{q}\right)}\|u(\cdot, s)\|_{L^{\infty}(\Omega)}^{a} d s \\
& \leq c_{1} m+c_{2} m^{1-a} K^{\frac{1}{p}}\left[c_{3} S_{1}^{a}+\frac{2 q}{q-2} S_{2}^{a}(T)\right],
\end{aligned}
$$

where $S_{2}(T):=\max \left\{\|u(\cdot, t)\|_{L^{\infty}(\Omega)} \mid t \in\left[t_{0}+1, T\right]\right\}$. In particular, with analogous arguments employed to derive the uniform bound for $S_{1}$, we can find $c_{5}>0$ such that $S_{2}(T) \leq c_{5}$. Finally, these estimates entail that $S(T):=\max \left\{S_{1}, S_{2}(T)\right\}$ is bounded, independently of $T$, proving (34), with, for instance, $C=\max \left\{c_{4}, \frac{c_{4}}{\tau}, c_{5}\right\}$.

In view of this crucial result, our final aim is to provide conditions capable of justifying eventual bounds for $\nabla w$ in $L^{p}(\Omega)$, with some $p>2$. This will be achieved by means of the forthcoming derivations, most of them tied to properties of the functional

$$
\mathcal{G}(t):=\mathcal{G}(u(\cdot, t), w(\cdot, t))=\frac{1}{2} \int_{\Omega}|\nabla w(\cdot, t)|^{2}+\int_{\Omega} H(u(\cdot, t)),
$$

defined for any $t>0$ and associated to the global classical solution $(u, w)$ of $(12)$, where

$$
H(\xi):=-\frac{1}{\chi} \int_{0}^{\xi} \int_{s}^{\infty} \frac{f^{\prime}(\sigma)}{\sigma} d \sigma d s .
$$

Further, let us remark that $\mathcal{G}$ generalizes the functional employed in [24] and [3] for $f(u) \equiv u$. 
Lemma 5.2 Assuming (A), (5) and (6), let $(u, w)$ be the global classical solution of problem (12) provided by Theorem 2.1 and (11). Then

$$
\int_{\Omega}|\nabla w|^{2} \leq 2 \mathcal{G}(u, w)+\frac{2 m^{\beta}|\Omega|^{1-\beta}}{\chi(1-\beta)} \quad \text { on }(0, \infty)
$$

and

$$
\mathcal{G}(u(\cdot, t), w(\cdot, t)) \leq \frac{1}{2} \int_{\Omega}|\nabla w(\cdot, t)|^{2} \quad \text { for all } t>0 .
$$

Moreover, we also have that

$$
\begin{aligned}
& \frac{d}{d t} \mathcal{G}(u, w)+\frac{1}{\chi} \int_{\Omega} \frac{f^{\prime}(u)|\nabla u|^{2}}{u} \\
& \quad+\frac{1}{2}\left(1-C_{G N} \int_{\Omega}|\nabla w|^{2}\right) \int_{\Omega}(\Delta w)^{2} \leq 0 \quad \text { on }(0, \infty),
\end{aligned}
$$

where $C_{G N}$ is the constant introduced in Lemma 3.2.

Proof Due to (5), the function $H$ is nonpositive on $(0, \infty)$ and can be estimated by

$$
-H(\xi) \leq \frac{1}{\chi} \int_{0}^{\xi} \int_{s}^{\infty} \beta \sigma^{\beta-2} d \sigma d s=\frac{1}{\chi(1-\beta)} \xi^{\beta} \quad \text { for all } \xi>0,
$$

so that Hölder's inequality implies

$$
\frac{1}{2} \int_{\Omega}|\nabla w|^{2}=\mathcal{G}(u, w)-\int_{\Omega} H(u) \leq \mathcal{G}(u, w)+\frac{m^{\beta}|\Omega|^{1-\beta}}{\chi(1-\beta)},
$$

which warrants (37), whilst (38) is an easy consequence of the definition of $\mathcal{G}$ and nonpositivity of $H$.

Additionally, thanks to the first equation of (12) we have that

$$
\begin{aligned}
\frac{d}{d t} \int_{\Omega} H(u) & =\int_{\Omega} H^{\prime}(u)(\Delta u+\chi \nabla \cdot(u \nabla w)) \\
& =-\int_{\Omega} H^{\prime \prime}(u)|\nabla u|^{2}-\chi \int_{\Omega} H^{\prime \prime}(u) u \nabla u \cdot \nabla w \\
& =-\frac{1}{\chi} \int_{\Omega} \frac{f^{\prime}(u)|\nabla u|^{2}}{u}-\int_{\Omega} f^{\prime}(u) \nabla u \cdot \nabla w \quad \text { on }(0, \infty) .
\end{aligned}
$$

Moreover, from the second equation of (12), the Young inequality and relation (15), we achieve on $(0, \infty)$ that

$$
\begin{aligned}
\frac{1}{2} \frac{d}{d t} \int_{\Omega}|\nabla w|^{2}+\int_{\Omega}(\Delta w)^{2} & =\int_{\Omega}|\nabla w|^{2} \Delta w+\int_{\Omega} f^{\prime}(u) \nabla u \cdot \nabla w \\
& \leq \frac{1}{2} \int_{\Omega}(\Delta w)^{2}+\frac{1}{2} \int_{\Omega}|\nabla w|^{4}+\int_{\Omega} f^{\prime}(u) \nabla u \cdot \nabla w \\
& \leq \frac{1}{2} \int_{\Omega}(\Delta w)^{2}+\frac{C_{G N}}{2} \int_{\Omega}|\nabla w|^{2} \int_{\Omega}(\Delta w)^{2}+\int_{\Omega} f^{\prime}(u) \nabla u \cdot \nabla w
\end{aligned}
$$

so that by adding the latest two relations we can conclude. 
The next result will be employed in the sequel to establish eventual boundedness of the term $\int_{\Omega}|\nabla w|^{2}$, such an estimate being strongly necessary to our purposes.

Lemma 5.3 Assuming (A), (5) and (6), let $(u, w)$ be the global classical solution of problem (12) provided by Theorem 2.1 and (11). Moreover, let $C_{G N}$ be the constant from Lemma 3.2. If there exists $t_{0} \geq 0$ such that the functional $\mathcal{G}$ defined in (36) satisfies

$$
\mathcal{G}\left(t_{0}\right)<\frac{1}{4 C_{G N}}-\frac{m^{\beta}|\Omega|^{\beta-1}}{\chi(1-\beta)},
$$

then

$$
\mathcal{G}^{\prime}(t) \leq 0 \text { for all } t>t_{0}
$$

Proof As in [24, Lemma 3.4], by taking into consideration (37) and assumption (40) we see that

$$
\begin{aligned}
\int_{\Omega}\left|\nabla w\left(\cdot, t_{0}\right)\right|^{2} \leq 2 \mathcal{G}\left(t_{0}\right)+2 \frac{m^{\beta}|\Omega|^{\beta-1}}{\chi(1-\beta)} & <\frac{2}{4 C_{G N}}-2 \frac{m^{\beta}|\Omega|^{\beta-1}}{\chi(1-\beta)}+2 \frac{m^{\beta}|\Omega|^{\beta-1}}{\chi(1-\beta)} \\
& =\frac{1}{2 C_{G N}},
\end{aligned}
$$

so that the set

$$
S:=\left\{t \geq\left. t_{0}\left|C_{G N} \int_{\Omega}\right| \nabla w(\cdot, \tau)\right|^{2}<\frac{1}{2} \text { for all } \tau \in\left(t_{0}, t\right)\right\}
$$

is not empty; more precisely, we aim to show that $T:=\sup S=\infty$. Indeed, if $T$ was finite, from the continuity of $t \mapsto \int_{\Omega}|\nabla w(\cdot, t)|^{2}$ we would necessarily have that

$$
C_{G N} \int_{\Omega}|\nabla w(\cdot, T)|^{2}=\frac{1}{2}
$$

On the other hand, from (39) and in view of the nonnegativity of $f^{\prime}$ due to (5) it is inferred that

$$
\mathcal{G}^{\prime}(t) \leq-\frac{1}{2}\left(1-C_{G N} \int_{\Omega}|\nabla w(\cdot, t)|^{2}\right) \int_{\Omega}|\Delta w(\cdot, t)|^{2} \leq-\frac{1}{4} \int_{\Omega}|\Delta w(\cdot, t)|^{2} \leq 0
$$

for all $t \in\left[t_{0}, T\right)$, so that $\mathcal{G}(T) \leq \mathcal{G}\left(t_{0}\right)$ and, again by virtue of (37) and (40),

$$
\int_{\Omega}|\nabla w(\cdot, T)|^{2} \leq 2 \mathcal{G}(T)+2 \frac{m^{\beta}|\Omega|^{\beta-1}}{\chi(1-\beta)} \leq 2 \mathcal{G}\left(t_{0}\right)+2 \frac{m^{\beta}|\Omega|^{\beta-1}}{\chi(1-\beta)}<\frac{1}{2 C_{G N}},
$$

which contradicts (41); then $T=\infty$ and the proof is given.

Lemma 5.4 Assuming (A) and (6), let $(u, w)$ be the global classical solution of problem (12) provided by Theorem 2.1 and (11). Then for any positive $\varepsilon_{1}$ we have that

$$
\frac{d}{d t} \int_{\Omega} u^{2}+\int_{\Omega}|\nabla u|^{2} \leq \varepsilon_{1} \int_{\Omega}|\nabla w|^{6}+D_{1}\left(\varepsilon_{1}\right) \int_{\Omega} u^{3} \quad \text { on }(0, \infty)
$$

where $D_{1}\left(\varepsilon_{1}\right)=\frac{\chi^{3}}{3}\left(6 \varepsilon_{1}\right)^{-\frac{1}{2}}$. 
Proof By multiplying the first equation of problem (12) by $u$, an integration by parts implies

$$
\frac{1}{2} \frac{d}{d t} \int_{\Omega} u^{2}+\int_{\Omega}|\nabla u|^{2}=-\chi \int_{\Omega} u \nabla u \cdot \nabla w \quad \text { on }(0, \infty) .
$$

The claim is obtained once we use in (43) that

$$
-\chi \int_{\Omega} u \nabla u \cdot \nabla w \leq \frac{1}{2} \int_{\Omega}|\nabla u|^{2}+\varepsilon_{1} \int_{\Omega}|\nabla w|^{6}+D_{1}\left(\varepsilon_{1}\right) \int_{\Omega} u^{3} \quad \text { on }(0, \infty),
$$

achieved thanks to two applications of the Young inequality, the first with exponents $\frac{1}{2}$ and $\frac{1}{2}$ and the second with $\frac{1}{3}$ and $\frac{2}{3}$.

The following results will all be aimed at controlling the size of $\int_{\Omega}|\nabla w|^{2}$ at large time $t$.

Lemma 5.5 Assuming (A) and (6), let $(u, w)$ be the global classical solution of problem (12) provided by Theorem 2.1 and (11). Then for any positive $\varepsilon_{2}$ we have that on $(0, \infty)$

$$
\begin{aligned}
\frac{d}{d t} \int_{\Omega}|\nabla w|^{4}+\left.\left.\frac{9}{16} \int_{\Omega}|\nabla| \nabla w\right|^{2}\right|^{2} \leq & \left(\frac{16}{9}+96 \varepsilon_{2}\right) \int_{\Omega}|\nabla w|^{6}+96 D_{2}\left(\varepsilon_{2}\right) \beta \int_{\Omega} u^{3} \\
& +C_{\partial \Omega}\left(\int_{\Omega}|\nabla w|^{2}\right)^{2}+96 D_{2}\left(\varepsilon_{2}\right)(1-\beta)|\Omega|,
\end{aligned}
$$

where $D_{2}\left(\varepsilon_{2}\right)=\frac{2}{3}\left(3 \varepsilon_{2}\right)^{-\frac{1}{2}}$ and $C_{\partial \Omega}$ is as in Lemma 3.3.

Proof By means of the identity $\Delta|\nabla w|^{2}=2 \nabla w \cdot \nabla \Delta w+2\left|D^{2} w\right|^{2}$ and using the second equation of (12) and its corresponding boundary conditions we can write

$$
\begin{aligned}
\frac{d}{d t} \int_{\Omega}|\nabla w|^{4}= & 4 \int_{\Omega}|\nabla w|^{2} \nabla w \cdot\left(\nabla \Delta w-\nabla|\nabla w|^{2}+\nabla f(u)\right) \\
= & 2 \int_{\Omega}|\nabla w|^{2} \Delta|\nabla w|^{2}-4 \int_{\Omega}\left|D^{2} w\right|^{2}|\nabla w|^{2} \\
& -4 \int_{\Omega}|\nabla w|^{2} \nabla w \cdot \nabla|\nabla w|^{2}+4 \int_{\Omega} f^{\prime}(u)|\nabla w|^{2} \nabla u \cdot \nabla w \quad \text { on }(0, \infty) .
\end{aligned}
$$

Now integration by parts gives

$$
\begin{aligned}
\frac{d}{d t} \int_{\Omega}|\nabla w|^{4}+\left.\left.2 \int_{\Omega}|\nabla| \nabla w\right|^{2}\right|^{2}+4 \int_{\Omega}\left|D^{2} w\right|^{2}|\nabla w|^{2} \\
\leq-4 \int_{\Omega}|\nabla w|^{2} \nabla w \cdot \nabla|\nabla w|^{2}-4 \int_{\Omega} f(u) \nabla w \cdot \nabla|\nabla w|^{2} \\
\quad-4 \int_{\Omega} f(u)|\nabla w|^{2} \Delta w+2 \int_{\partial \Omega}|\nabla w|^{2} \frac{\partial|\nabla w|^{2}}{\partial v} \quad \text { on }(0, \infty),
\end{aligned}
$$

where, according to Lemma 3.3, we can estimate

$$
2 \int_{\partial \Omega}|\nabla w|^{2} \frac{\partial|\nabla w|^{2}}{\partial v} \leq\left.\left.\frac{1}{16} \int_{\Omega}|\nabla| \nabla w\right|^{2}\right|^{2}+C_{\partial \Omega}\left(\int_{\Omega}|\nabla w|^{2}\right)^{2} \quad \text { on }(0, \infty) .
$$


Subsequently the Young inequality produces

$$
-4 \int_{\Omega}|\nabla w|^{2} \nabla w \cdot \nabla|\nabla w|^{2} \leq\left.\left.\frac{9}{4} \int_{\Omega}|\nabla| \nabla w\right|^{2}\right|^{2}+\frac{16}{9} \int_{\Omega}|\nabla w|^{6} \quad \text { on }(0, \infty),
$$

and

$$
\begin{aligned}
& -4 \int_{\Omega} f(u) \nabla w \cdot \nabla|\nabla w|^{2}-4 \int_{\Omega} f(u)|\nabla w|^{2} \Delta w \\
& \leq\left.\left.\frac{1}{16} \int_{\Omega}|\nabla| \nabla w\right|^{2}\right|^{2}+64 \int_{\Omega} u^{2 \beta}|\nabla w|^{2}+\frac{1}{8} \int_{\Omega}|\nabla w|^{2}|\Delta w|^{2}+32 \int_{\Omega} u^{2 \beta}|\nabla w|^{2} \\
& \leq\left.\left.\frac{1}{16} \int_{\Omega}|\nabla| \nabla w\right|^{2}\right|^{2}+\frac{1}{4} \int_{\Omega}\left|D^{2} w\right|^{2}|\nabla w|^{2}+96 \int_{\Omega} u^{2 \beta}|\nabla w|^{2} \quad \text { on }(0, \infty),
\end{aligned}
$$

where we have used the pointwise relation $|\Delta w|^{2} \leq 2\left|D^{2} w\right|^{2}$, valid throughout $\Omega$, and, of course, (4).

On the other hand, again two applications of the Young inequality for any $\varepsilon_{2}>0$ yield

$$
\begin{aligned}
\int_{\Omega} u^{2 \beta}|\nabla w|^{2} \leq & \varepsilon_{2} \int_{\Omega}|\nabla w|^{6}+D_{2}\left(\varepsilon_{2}\right) \int_{\Omega} u^{3 \beta} \leq \varepsilon_{2} \int_{\Omega}|\nabla w|^{6}+D_{2}\left(\varepsilon_{2}\right) \beta \int_{\Omega} u^{3} \\
& +D_{2}\left(\varepsilon_{2}\right)(1-\beta)|\Omega| \quad \text { on }(0, \infty)
\end{aligned}
$$

with $D_{2}\left(\varepsilon_{2}\right)=\frac{2}{3}\left(3 \varepsilon_{2}\right)^{-\frac{1}{2}}$.

Finally, by plugging (46), (47), (48) and (49) into (45), and in view of the relation

$$
\left.\left.|\nabla| \nabla w\right|^{2}\right|^{2}=4\left|D^{2} w \nabla w\right|^{2} \leq 4\left|D^{2} w\right|^{2}|\nabla w|^{2}
$$

we readily have the claim.

Lemma 5.6 Assuming (A) and (6), let $(u, w)$ be the global classical solution of problem (12) provided by Theorem 2.1 and (11). If for some $M>0$ and $t_{0} \geq 0$

$$
\int_{\Omega}|\nabla w(\cdot, t)|^{2} \leq M \quad \text { for all } t>t_{0}
$$

then we have that

$$
\begin{aligned}
& \frac{d}{d t}\left(\int_{\Omega} u^{2}+\int_{\Omega}|\nabla w|^{4}\right)+\left(1-8 C_{G N}^{3} m\left(D_{1}\left(\varepsilon_{1}\right)+96 \beta D_{2}\left(\varepsilon_{2}\right)\right)\right) \int_{\Omega}|\nabla u|^{2} \\
& \quad+\left.\left.\left(\frac{9}{16}-2\left(\varepsilon_{1}+\frac{16}{9}+96 \varepsilon_{2}\right) C_{G N} M\right) \int_{\Omega}|\nabla| \nabla w\right|^{2}\right|^{2} \\
& \leq C_{\partial \Omega} M^{2}+96 D_{2}\left(\varepsilon_{2}\right)(1-\beta)|\Omega|+8 m^{3} C_{G N}^{3}\left(D_{1}\left(\varepsilon_{1}\right)+96 \beta D_{2}\left(\varepsilon_{2}\right)\right) \\
& \quad+\left(\varepsilon_{1}+\frac{16}{9}+96 \varepsilon_{2}\right) C_{G N}^{2} M^{3} \text { on }\left(t_{0}, \infty\right),
\end{aligned}
$$

where $C_{G N}$ is the constant introduced in Lemma 3.2, $\varepsilon_{1}$ and $\varepsilon_{2}$ are arbitrary positive constants and $D_{1}\left(\varepsilon_{1}\right)$ and $D_{2}\left(\varepsilon_{2}\right)$ have been defined in Lemmas 5.4 and 5.5 and where $m=\int_{\Omega} u_{0}$. 
Proof By adding the inequalities (42) and (44), both valid on $(0, \infty)$, we get

$$
\begin{aligned}
& \frac{d}{d t}\left(\int_{\Omega} u^{2}+\int_{\Omega}|\nabla w|^{4}\right)+\int_{\Omega}|\nabla u|^{2}+\left.\left.\frac{9}{16} \int_{\Omega}|\nabla| \nabla w\right|^{2}\right|^{2} \\
& \leq\left(\varepsilon_{1}+\frac{16}{9}+96 \varepsilon_{2}\right) \int_{\Omega}|\nabla w|^{6}+\left(D_{1}\left(\varepsilon_{1}\right)+96 \beta D_{2}\left(\varepsilon_{2}\right)\right) \int_{\Omega} u^{3} \\
& \quad+C_{\partial \Omega} M^{2}+96 D_{2}\left(\varepsilon_{2}\right)(1-\beta)|\Omega| \text { on }(0, \infty) .
\end{aligned}
$$

Now, we apply (14) to achieve throughout $(0, \infty)$

$$
\begin{aligned}
\int_{\Omega}|\nabla w|^{6} & =\left\||\nabla w|^{2}\right\|_{L^{3}(\Omega)}^{3} \leq C_{G N}\left\||\nabla w|^{2}\right\|_{L^{1}(\Omega)}\left\||\nabla w|^{2}\right\|_{W^{1,2}(\Omega)}^{2} \\
& \leq C_{G N} \int_{\Omega}|\nabla w|^{2} \int_{\Omega}|\nabla w|^{4}+\left.\left.C_{G N} \int_{\Omega}|\nabla w|^{2} \int_{\Omega}|\nabla| \nabla w\right|^{2}\right|^{2},
\end{aligned}
$$

and using

$$
\int_{\Omega}|\nabla w|^{4} \leq\left(\int_{\Omega}|\nabla w|^{6}\right)^{\frac{1}{2}}\left(\int_{\Omega}|\nabla w|^{2}\right)^{\frac{1}{2}} \text { on }(0, \infty),
$$

we obtain through the Young inequality and assumption (50)

$$
\begin{aligned}
\int_{\Omega}|\nabla w|^{6} & \leq C_{G N}\left(\int_{\Omega}|\nabla w|^{6}\right)^{\frac{1}{2}}\left(\int_{\Omega}|\nabla w|^{2}\right)^{\frac{3}{2}}+\left.\left.C_{G N} \int_{\Omega}|\nabla w|^{2} \int_{\Omega}|\nabla| \nabla w\right|^{2}\right|^{2} \\
& \leq \frac{1}{2} \int_{\Omega}|\nabla w|^{6}+\frac{C_{G N}^{2}}{2}\left(\int_{\Omega}|\nabla w|^{2}\right)^{3}+\left.\left.C_{G N} \int_{\Omega}|\nabla w|^{2} \int_{\Omega}|\nabla| \nabla w\right|^{2}\right|^{2} \\
& \leq \frac{1}{2} \int_{\Omega}|\nabla w|^{6}+\frac{C_{G N}^{2} M^{3}}{2}+\left.\left.C_{G N} M \int_{\Omega}|\nabla| \nabla w\right|^{2}\right|^{2} \quad \text { on }\left(t_{0}, \infty\right) .
\end{aligned}
$$

Turning our attention to the term $\int_{\Omega} u^{3}$, the Gagliardo-Nirenberg inequality (13) with the particular choice $\mathfrak{p}=3, \mathfrak{s}=\mathfrak{q}=1$ and $\theta=\frac{2}{3}<1$, gives in conjunction with (17),

$$
\begin{aligned}
\int_{\Omega} u^{3} & =\|u\|_{L^{3}(\Omega)}^{3} \leq\left[C_{G N}\left(\|\nabla u\|_{L^{2}(\Omega)}^{\theta}\|u\|_{L^{1}(\Omega)}^{1-\theta}+\|u\|_{L^{1}(\Omega)}\right)\right]^{3} \\
& \leq\left(8 C_{G N}^{3}\right)\left(m \int_{\Omega}|\nabla u|^{2}+m^{3}\right) \quad \text { on }(0, \infty)
\end{aligned}
$$

where we also considered the mass conservation property, i.e. $\int_{\Omega} u=m$. Finally, by using (53) and (54), (52) reads exactly as in (51).

In the next result we shall show uniform-in-time boundedness of the $L^{4}(\Omega)$-norm of $\nabla w$ beyond some time, which will be used in order to obtain eventual boundedness of $u$. This is possible through a smallness assumption on $m$.

Lemma 5.7 Assume (A). For any $M \in\left(0, \frac{9}{17.32 C_{G N}}\right)$, it is possible to find $\gamma>0$ such that if a global solution $(u, w)$ of problem (12) emanates from initial data as in (6) and (11) and 
fulfilling $\int_{\Omega} u_{0}=: m<\bar{m}:=\frac{1}{16 \gamma C_{G N}^{3}}$ and also satisfies (50) of Lemma 5.6 for some $t_{0}>0$, then it has the following property: For any $\tau>0$ there exists a positive constant $K$ such that

$$
\int_{\Omega}|\nabla w(\cdot, t)|^{4} \leq K \quad \text { for all } t>t_{0}+\tau
$$

Proof Let $D_{1}\left(\varepsilon_{1}\right)$ and $D_{2}\left(\varepsilon_{2}\right)$ be the $\varepsilon$-dependent functions defined in Lemmas 5.4 and 5.5. Since $M \in\left(0, \frac{9}{17 \cdot 32 C_{G N}}\right)$, by choosing

$$
\varepsilon_{1}=\frac{1}{32 M C_{G N}}-\frac{17}{9}>0, \quad \varepsilon_{2}=\frac{1}{96 \cdot 9} \quad \text { and } \quad \gamma=D_{1}\left(\varepsilon_{1}\right)+96 \beta D_{2}\left(\varepsilon_{2}\right)
$$

we have that, in view of the assumption $\int_{\Omega} u_{0}=m<\bar{m}=\frac{1}{16 \gamma C_{G N}^{3}}$,

$$
2\left(\varepsilon_{1}+\frac{16}{9}+96 \varepsilon_{2}\right) C_{G N} M=\frac{1}{16} \text { and } 8 C_{G N}^{3} m \gamma \leq \frac{1}{2} .
$$

Hence, through (50) of Lemma 5.6, inequality (51) reads

$$
\frac{d}{d t}\left(\int_{\Omega} u^{2}+\int_{\Omega}|\nabla w|^{4}\right)+\frac{1}{2} \int_{\Omega}|\nabla u|^{2}+\left.\left.\frac{1}{2} \int_{\Omega}|\nabla| \nabla w\right|^{2}\right|^{2} \leq c_{4} \quad \text { for all } t>t_{0},
$$

with $c_{4}=C_{\partial \Omega} M^{2}+96 D_{2}\left(\varepsilon_{2}\right)(1-\beta)|\Omega|+8 \bar{m}^{3} C_{G N}^{3} \gamma+C_{G N} M^{2} / 16$.

Now we are in the favourable position to control the integrals involving $|\nabla u|^{2}$ and $\left.\left.|\nabla| \nabla w\right|^{2}\right|^{2}$ by using again (13) for $\mathfrak{p}=2, \mathfrak{q}=\mathfrak{s}=1$ and $\theta=\frac{1}{2}$, which shows that

$$
\|f\|_{L^{2}}^{4} \leq\left(2 C_{G N}\right)^{4}\left(\|\nabla f\|_{L^{2}}^{2}\|f\|_{L^{1}}^{2}+\|f\|_{L^{1}}^{4}\right)
$$

and hence for $f \in L^{4}(\Omega)$ with $\nabla f \in L^{2}(\Omega)$

$$
-\frac{1}{2}\|\nabla f\|_{L^{2}}^{2} \leq-\frac{\|f\|_{L^{2}}^{4}}{2\left(2 C_{G N}\right)^{4}\|f\|_{L^{1}}^{2}}+\frac{\|f\|_{L^{1}}^{2}}{2}
$$

In particular, if we take into account the mass conservation property $\int_{\Omega} u=\bar{m}$, for all $t>0$ we can write

$$
-\frac{1}{2} \int_{\Omega}|\nabla u(\cdot, t)|^{2} \leq-\frac{1}{2\left(2 C_{G N}\right)^{4} m^{2}}\left(\int_{\Omega} u^{2}(\cdot, t)\right)^{2}+\frac{m^{2}}{2}
$$

and similarly, by relying on (50), from (56) we arrive at

$$
-\left.\left.\frac{1}{2} \int_{\Omega}|\nabla| \nabla w(\cdot, t)\right|^{2}\right|^{2} \leq-\frac{1}{2\left(2 C_{G N}\right)^{4} M^{2}}\left(\int_{\Omega}|\nabla w(\cdot, t)|^{4}\right)^{2}+\frac{M^{2}}{2} \quad \text { for all } t>0 .
$$

From these bounds, and setting $\Phi(t):=\int_{\Omega} u^{2}+\int_{\Omega}|\nabla w|^{4}$, the inequality (55) and $m<\bar{m}$ imply that $\Phi$ is a sub-solution of the ordinary differential equation

$$
\Psi^{\prime}(t)=-c_{5} \Psi^{2}(t)+c_{6} \text { for all } t>t_{0},
$$

with $c_{5}=\frac{1}{4\left(2 C_{G N}\right)^{4}} \max \left\{\bar{m}^{2}, M^{2}\right\}^{-2}$ and $c_{6}=c_{4}+\frac{M^{2}+\bar{m}^{2}}{2}$. 
By considering the function $\bar{\Phi}(t):=\frac{1}{c_{5}\left(t-t_{0}\right)}+\sqrt{\frac{c_{6}}{c_{5}}}, t \in\left(t_{0}, \infty\right)$, we see that

$$
\bar{\Phi}^{\prime}(t)+c_{5} \bar{\Phi}^{2}(t)-c_{6}=2 \sqrt{\frac{c_{6}}{c_{5}}}\left(t-t_{0}\right)^{-1} \geq 0 \quad \text { for all } t>t_{0},
$$

so that $\bar{\Phi}$ is a super-solution of (57) such that $\bar{\Phi}(t) \nearrow+\infty$ as $t \searrow t_{0}$. Subsequently an ODE comparison reasoning leads to $\Phi(t) \leq \bar{\Phi}(t)$ for all $t>t_{0}$ and in particular we have

$$
\int_{\Omega}|\nabla w(\cdot, t)|^{4} \leq \Phi(t) \leq \bar{\Phi}\left(t_{0}+\tau\right)=\frac{1}{c_{5} \tau}+\sqrt{\frac{c_{6}}{c_{5}}} \quad \text { for all } t \geq t_{0}+\tau .
$$

Lemma 5.8 Under the assumption (A), for any $\Gamma>0$, it is possible to find $\hat{m}(\Gamma)>0$ such that for any initial data $\left(u_{0}, v_{0}\right)$ as in (6) and also fulfilling $\int_{\Omega} u_{0} \leq \hat{m}$, there is $t_{*}>0$ such that the corresponding global classical solution $(u, w)$ of problem (12) satisfies

$$
\int_{\Omega}\left|\nabla w\left(\cdot, t_{*}\right)\right|^{2} \leq \Gamma
$$

Proof Given $m>0$, we let $L_{1}(m)$ be as in Lemma 4.1 and

$$
C_{1}(m):=C_{1}\left(m, L_{1}(m)\right)=m\left(2 C_{G N}\right)^{4}\left(L_{1}(m)+m\right)
$$

the corresponding constant introduced in Lemma 3.4. Since $L_{1}(m)$ remains bounded in a neighbourhood of $m=0, C_{1}(m) \searrow 0$ as $m \searrow 0$, so, corresponding to $\Gamma>0$, we then choose $\hat{m}=\hat{m}(\Gamma)>0$ such that $C_{1}(m)<2^{-1-\frac{2}{\beta}}|\Omega|^{1-\frac{2}{\beta}} \Gamma^{\frac{2}{\beta}}$ for all $m \in(0, \hat{m})$. From integrating the second equation of (12) over $(0, t) \times \Omega$, (4) and Hölder's inequality, we obtain for any $t>0$ that

$$
\int_{\Omega} w-\int_{\Omega} w_{0}+\int_{0}^{t} \int_{\Omega}|\nabla w|^{2}=\int_{0}^{t} \int_{\Omega} f(u) \leq(t|\Omega|)^{1-\frac{\beta}{2}}\left(\int_{0}^{t} \int_{\Omega} u^{2}\right)^{\frac{\beta}{2}} .
$$

Owing to the estimate $\int_{0}^{t} \int_{\Omega} u^{2} \leq C_{1} t+C_{2}$ of Lemmata 4.1 and 3.4 combined, where $C_{2}:=$ $C_{2}\left(m, L_{2}\left(u_{0}, w_{0}\right)\right)$, due to the nonnegativity of $w$ we deduce from (58) and (17) that

$$
\int_{0}^{t} \int_{\Omega}|\nabla w|^{2} \leq|\Omega|^{1-\frac{\beta}{2}} t^{1-\frac{\beta}{2}}\left(\left(2 C_{1}(\hat{m}) t\right)^{\frac{\beta}{2}}+\left(2 C_{2}\right)^{\frac{\beta}{2}}\right)+\int_{\Omega} w_{0} \quad \text { for all } t>0,
$$

and for any $t>0$ the average theorem establishes the existence of a time $t_{*} \in\left(\frac{t}{2}, t\right)$ such that

$$
\begin{aligned}
\int_{\Omega}\left|\nabla w\left(\cdot, t_{*}\right)\right|^{2} & =\frac{2}{t} \int_{\frac{t}{2}}^{t} \int_{\Omega}|\nabla w|^{2} \leq \frac{2}{t} \int_{0}^{t} \int_{\Omega}|\nabla w|^{2} \\
& \leq 2^{1+\frac{\beta}{2}}|\Omega|^{1-\frac{\beta}{2}} C_{1}^{\frac{\beta}{2}}(\hat{m})+2^{\frac{\beta}{2}+1}|\Omega|^{1-\frac{\beta}{2}} C_{2}^{\frac{\beta}{2}} t^{-\frac{\beta}{2}}+\frac{2}{t} \int_{\Omega} w_{0} .
\end{aligned}
$$

According to our choice of $\hat{m}$, for any initial data with $\int_{\Omega} u_{0} \leq \hat{m}$ it is therefore apparently possible to choose $t$ sufficiently large so as to conclude the existence of $t_{*}$ satisfying

$$
\int_{\Omega}\left|\nabla w\left(\cdot, t_{*}\right)\right|^{2} \leq \Gamma,
$$

and the proof is concluded. 
With the above derived information, and assuming a suitable smallness condition on $m=\int_{\Omega} u \equiv \int_{\Omega} u_{0}$, we can now ensure eventual boundedness of the spatial $L^{2}$-norm of $\nabla w$. Precisely we have

Lemma 5.9 Assume (A) and (5). For any $M>0$, there is $m_{*}>0$ such that for any initial data $\left(u_{0}, v_{0}\right)$ as in (6) and also fulfilling $\int_{\Omega} u_{0} \leq m_{*}$, there is $t_{*}>0$ such that the corresponding global classical solution $(u, w)$ of problem (12) provided by Theorem 2.1 and (11) satisfies

$$
\int_{\Omega}|\nabla w(\cdot, t)|^{2} \leq M \quad \text { for all } t \geq t_{*}
$$

Proof Without loss of generality, we assume $M \in\left(0, \frac{9}{17 \cdot 32 C_{G N}}\right)$ and with $\bar{m}$ from Lemma 5.7, let us choose some $\tilde{m}>0$ such that

$$
\tilde{m}<\min \left\{\left(\frac{\chi(1-\beta)}{4|\Omega|^{\beta-1} C_{G N}}\right)^{\frac{1}{\beta}},\left(\frac{M \chi(1-\beta)}{4|\Omega|^{1-\beta}}\right)^{\frac{1}{\beta}}, \bar{m}\right\},
$$

which in particular implies

$$
\frac{1}{4 C_{G N}}-\frac{\tilde{m}^{\beta}|\Omega|^{\beta-1}}{\chi(1-\beta)}>0
$$

Now let us pick

$$
0<\Gamma<\min \left\{\frac{2}{4 C_{G N}}-\frac{2 \tilde{m}^{\beta}|\Omega|^{\beta-1}}{\chi(1-\beta)}, \frac{M}{2}\right\} .
$$

In light of Lemma 5.8, we can find $\hat{m}=\hat{m}(\Gamma)$ so that for any solution emanating from initial data with $\int_{\Omega} u_{0}<\hat{m}$ there is $t_{*}>0$ so that

$$
\int_{\Omega}\left|\nabla w\left(\cdot, t_{*}\right)\right|^{2} \leq \Gamma
$$

Letting $m_{*}:=\min \{\hat{m}, \tilde{m}\}$ and assuming that $\int_{\Omega} u_{0}=m \leq m_{*}$ and $t_{*}$ is such that (62) holds, thanks to (38) we have that

$$
\mathcal{G}\left(t_{*}\right) \leq \frac{1}{2} \int_{\Omega}\left|\nabla w\left(\cdot, t_{*}\right)\right|^{2} \leq \frac{\Gamma}{2}<\frac{1}{4 C_{G N}}-\frac{m_{*}^{\beta}|\Omega|^{\beta-1}}{\chi(1-\beta)} .
$$

We are now in the position to apply Lemma 5.3 and conclude that $\mathcal{G}^{\prime}(t) \leq 0$ for all $t>t_{*}$, which subsequently provides that $\mathcal{G}(t) \leq \mathcal{G}\left(t_{*}\right)$ for all $t \geq t_{*}$. Thereafter, from $m \leq m_{*}$ and (37) of Lemma 5.2 we have that

$$
\int_{\Omega}|\nabla w(\cdot, t)|^{2} \leq 2 \mathcal{G}(t)+\frac{2 m_{*}^{\beta}|\Omega|^{1-\beta}}{\chi(1-\beta)} \quad \text { for all } t>0,
$$

i.e. through (38) and (60)-(62)

$$
\begin{aligned}
\int_{\Omega}|\nabla w(\cdot, t)|^{2} & \leq 2 \mathcal{G}\left(t_{*}\right)+\frac{2 m_{*}^{\beta}|\Omega|^{1-\beta}}{\chi(1-\beta)} \\
& \leq \int_{\Omega}\left|\nabla w\left(\cdot, t_{*}\right)\right|^{2}+\frac{2 m_{*}^{\beta}|\Omega|^{1-\beta}}{\chi(1-\beta)} \leq M \quad \text { for all } t \geq t_{*} .
\end{aligned}
$$


Proof of Theorem 2.2 Let $m_{*}>0$ be the value introduced in Lemma 5.9 and let $(u, w)$ be the global classical solution of problem (3) provided by Theorem 2.1 and (11), and emanating from initial data $\left(u_{0}, v_{0}\right)$ as in (6) and such that $\int_{\Omega} u_{0} \leq m_{*}$. By virtue of Lemma 5.9, we can find $t_{*} \in(0, \infty)$ such that relation (59) holds with $M \in\left(0, \frac{9}{17 \cdot 32 C_{G N}}\right)$ and Lemma 5.7 becomes applicable so that with some $K>0$

$$
\int_{\Omega}|\nabla w(\cdot, t)|^{4} \leq K \quad \text { for all } t>t_{*}+1
$$

Finally Lemma 5.1 with the choice $p=4, t_{0}=t_{*}+1$ and $\tau=1$ provides the boundedness of $u$ in $\left(t_{*}+2, \infty\right)$. Due to continuity and hence boundedness of $u$ in $\bar{\Omega} \times\left[0, t_{*}+2\right]$, this concludes the proof.

Acknowledgements GV is member of the Gruppo Nazionale per l'Analisi Matematica, la Probabilità e le loro Applicazioni (GNAMPA) of the Istituto Nazionale di Alta Matematica (INdAM), gratefully acknowledges the Italian Ministry of Education, University and Research (MIUR) for the financial support of Scientific Project "Smart Cities and Communities and Social Innovation-ILEARNTV anywhere, anytimeSCN_00307" and is partially supported by the research project Integro-differential Equations and Non-Local Problems, funded by Fondazione di Sardegna (2017). This work was initiated while JL was visiting the Università di Cagliari, in the framework of the project "Blow-up and global existence of solutions to Keller-Segel type systems modelling chemotaxis" (INdAM-GNAMPA Project 2016). He is grateful for the hospitality.

Publisher's Note Springer Nature remains neutral with regard to jurisdictional claims in published maps and institutional affiliations.

\section{References}

1. Bellomo, N., Bellouquid, A., Tao, Y., Winkler, M.: Toward a mathematical theory of Keller-Segel models of pattern formation in biological tissues. Math. Models Methods Appl. Sci. 25(09), 1663-1763 (2015)

2. Biler, P.: Global solutions to some parabolic-elliptic systems of chemotaxis. Adv. Math. Sci. Appl. 9(1), 347-359 (1999)

3. Black, T.: Eventual smoothness of generalized solutions to a singular chemotaxis-Stokes system J. Differ. Equ. 265(5), 2296-2339 (2018)

4. Cao, X., Lankeit, J.: Global classical small-data solutions for a three-dimensional chemotaxis NavierStokes system involving matrix-valued sensitivities. Calc. Var. Partial Differ. Equ. 55(4), 107 (2016)

5. Duan, R., Lorz, A., Markowich, P.: Global solutions to the coupled chemotaxis-fluid equations. Commun. Partial Differ. Equ. 35(9), 1635-1673 (2010)

6. Fujie, K.: Boundedness in a fully parabolic chemotaxis system with singular sensitivity. J. Math. Anal. Appl. 424(1), 675-684 (2015)

7. Fujie, K., Senba, T.: A sufficient condition of sensitivity functions for boundedness of solutions to a parabolic-parabolic chemotaxis system. Nonlinearity 31(4), 1639 (2018)

8. Horstmann, D.: From 1970 until present: the Keller-Segel model in chemotaxis and its consequences I. Jahresber. Dtsch. Math.-Ver. 105(3), 103-165 (2003)

9. Ishida, S., Seki, K., Yokota, T.: Boundedness in quasilinear Keller-Segel systems of parabolic-parabolic type on non-convex bounded domains. J. Differ. Equ. 256(8), 2993-3010 (2014)

10. Keller, E.F., Segel, L.A.: Initiation of slime mold aggregation viewed as an instability. J. Theor. Biol. 26(3), 399-415 (1970)

11. Keller, E.F., Segel, L.A.: Traveling bands of chemotactic bacteria: a theoretical analysis. J. Theor. Biol. 30(2), 235-248 (1971)

12. Lankeit, J.: A new approach toward boundedness in a two-dimensional parabolic chemotaxis system with singular sensitivity. Math. Methods Appl. Sci. 39(3), 394-404 (2016)

13. Lankeit, J.: Locally bounded global solutions to a chemotaxis consumption model with singular sensitivity and nonlinear diffusion. J. Differ. Equ. 262(7), 4052-4084 (2017)

14. Lankeit, E., Lankeit, J.: Classical solutions to a logistic chemotaxis model with singular sensitivity and signal absorption. Nonlinear Anal., Real World Appl. 46, 421-445 (2019)

15. Lankeit, J., Winkler, M.: A generalized solution concept for the Keller-Segel system with logarithmic sensitivity: global solvability for large nonradial data. Nonlinear Differ. Equ. Appl. 24(4), 49 (2017) 
16. Liu, D.: Global classical solution to a chemotaxis consumption model with singular sensitivity. Nonlinear Anal., Real World Appl. 41, 497-508 (2018)

17. Liu, J.-G., Lorz, A.: A coupled chemotaxis-fluid model: global existence. Ann. Inst. Henri Poincaré, Anal. Non Linéaire 28(5), 643-652 (2011)

18. Nagai, T., Senba, T.: Global existence and blow-up of radial solutions to a parabolic-elliptic system of chemotaxis. Adv. Math. Sci. Appl. 8, 145-156 (1998)

19. Nirenberg, L.: On elliptic partial differential equations. Ann. Sc. Norm. Super. Pisa, Cl. Sci. (3) 2(13), 115-162 (1959)

20. Wang, Z.-A.: Mathematics of traveling waves in chemotaxis-review paper. Discrete Contin. Dyn. Syst., Ser. B 18(3), 601-641 (2013)

21. Wang, Y.: Global large-data generalized solutions in a two-dimensional chemotaxis-Stokes system with singular sensitivity. Bound. Value Probl. 2016, 177 (2016)

22. Wang, Z.-A., Xiang, Z., Yu, P.: Asymptotic dynamics on a singular chemotaxis system modeling onset of tumor angiogenesis. J. Differ. Equ. 260(3), 2225-2258 (2016)

23. Weibull, C.: Movement. In: The Bacteria, vol. I, pp. 153-205. Academic Press, New York (1960)

24. Winkler, M.: The two-dimensional Keller-Segel system with singular sensitivity and signal absorption: eventual smoothness and equilibration of small-mass solutions. Preprint

25. Winkler, M.: Aggregation vs. global diffusive behavior in the higher-dimensional Keller-Segel model. J. Differ. Equ. 248(12), 2889-2905 (2010)

26. Winkler, M.: The two-dimensional Keller-Segel system with singular sensitivity and signal absorption: global large-data solutions and their relaxation properties. Math. Models Methods Appl. Sci. 26(05), 987-1024 (2016)

27. Winkler, M.: Renormalized radial large-data solutions to the higher-dimensional Keller-Segel system with singular sensitivity and signal absorption. J. Differ. Equ. 264(3), 2310-2350 (2018)

28. Zhao, X., Zheng, S.: Global existence and asymptotic behavior to a chemotaxis-consumption system with singular sensitivity and logistic source. Nonlinear Anal., Real World Appl. 42, 120-139 (2018) 